Notas Clínicas

\title{
Sarcoma de Kaposi epidémico mucoso: aportación de un caso y revisión de la literatura
}

F. J. Andreu Martínez, J. M. Martínez Mateu

\section{Resumen}

- Propósito: Presentamos un caso clínico de sarcoma de Kaposi epidémico de laringe, con revisión y discusión de la literatura.

- Material y métodos: Paciente varón de 37 años, con antecedentes de SIDA y con clínica de disfonía y diagnosticado de Sarcoma de Kaposi epidémico laríngeo. Se realizó tratamiento con radioterapia.

- Resultados y conclusiones: Tras 3 años del tratamiento, el paciente se encuentra con la lesión laringea controlada. La radioterapia es un tratamiento paliativo de elección en el sarcoma de Kaposi epidémico mucoso.

Palabras clave:

SIDA. Sarcoma de Kaposi Epidémico. Radioterapia. Quimioterapia. HAART.

Oncología, 2005; 28 (5):239-243 


\section{Summary}

- Purpose: We present a clinical case of epidemic Kaposi's sarcoma of the larynx, make a review, and discuss the literature

- Material and methods: A 37 year old male with AIDS and hoarseness diagnosed of epidemic Kaposi's sarcoma of the larynx. He was treated with radiotherapy.

- Results and conclusions: Three years after radiotherapy, the patient is free of laryngeal disease. Radiation therapy is the paliative treatment of choice for AIDS-associated epidemic mucous Kaposi's sarcoma.

Key words: AIDS. Epidemic Kaposi’s sarcoma. Radiotherapy. Chemotherapy. HAART.

\section{Introducción}

En 1872 Moritz Kaposi describió 5 pacientes con lesiones rojo-violáceas que el denominó "sarcoma hemorrágico idiopático múltiple", posteriormente en 1912 Sternberg sugirió el epónimo de Sarcoma de Kaposi (SK), desde entonces diferentes formas de la enfermedad han podido ser identificadas ${ }^{1}$.

El Sarcoma de Kaposi Epidémico (SKE), fue descrito en los primeros años de la década de los 80 y es más frecuente, en varones homosexuales o bisexuales con SIDA, así como en mujeres que adquirían la enfermedad de hombres bisexuales (hasta un $25 \%)$, que en otros grupos de riesgo $(1-5 \%)^{2,3}$.

\section{Caso clínico}

Varón de 37 años de edad, con antecedentes personales de fumador de 12 años/paquete, consumidor habitual de cocaína y drogas sintéticas (anfetaminas, speed,...) desde los 25 años de edad, enolismo $<80$ gr. etanol/día. Homosexual fue diagnosticado de SIDA en Noviembre de 2001 (estadio C3), según los criterios de la CDC (neumonía por P. carinni y recuento de linfocitos <200 CD4/ $\mu \mathrm{L}$ ). El estudio de subpoblaciones linfoides en sangre periférica en aquella fecha mostró lo siguientes resultados CD4: 4,5\% (81), CD8: 71,7\% (1290) CD4/CD8: 0,06 у carga viral HIV: 19.200 copias RNA/ml. Con estos datos inició Tratamiento Antiretroviral de Alta Eficacia (HAART), utilizando 2 inhibidores de la transcriptasa inversa, zidovudina y lamivudina, más un inhibidor de la proteasa, abacavir, los cuales ha ido cambiando y combinando por otros fármacos de la misma familia (efavirenz, estavudina, ritonavir). En Diciembre 2001 acudió a Urgencias por presentar disfonía de 15 días de evolución, a la exploración ORL destacaba la existencia de una rinitis seca-costrosa anterior y bilateral compatible con rinitis por inhalación de cocaína, candidiasis oral y a la laringoscopia indirecta, lesión violácea localizada en banda ventricular y repliegue aritenoepiglótico izdo., movilidad de la cuerda vocal y aritenoides izdo. disminuida (Fig. 1). Ante la sospecha clínica de sarcoma de Kaposi, y dada las posibles complicaciones no se realizó una biopsia de la lesión, no obstante, se practicaron broncoscopia que informó de la existencia a nivel laríngeo de placas de color rojo intenso que también estaban localizadas en la porción

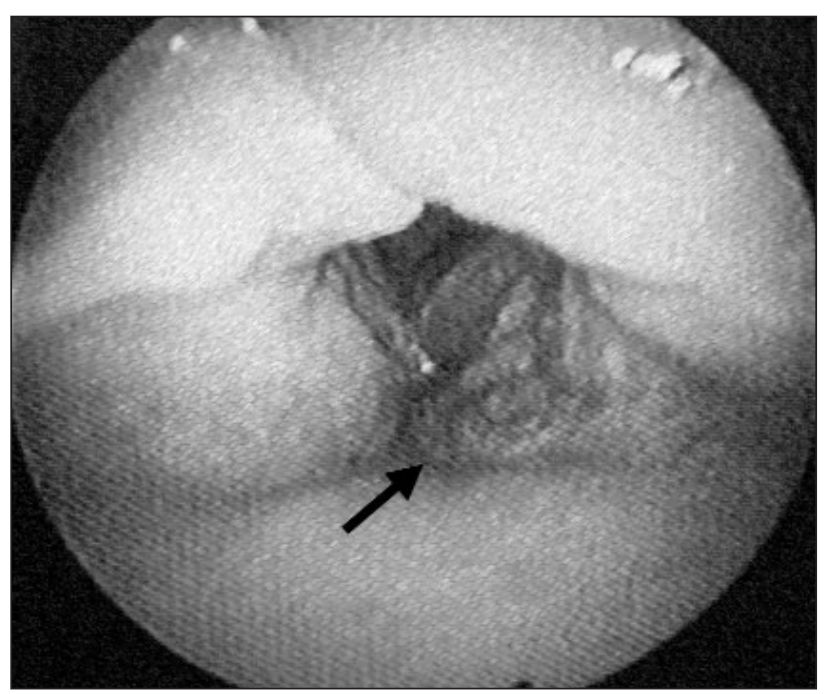

Figura 1. Laringoscopia directa donde se visualiza la lesión en banda y repliegue aritenoepiglótico izquierdo. 
más superior de la traquea y que igualmente eran compatibles con la presencia de sarcoma de Kaposi a dicho nivel; gastroscopia que informó de la no existencia de lesiones y colonoscopia que informó de la existencia de hemorroides grado II/IV con discreta estenosis del canal anal.

Con el diagnóstico clínico de sarcoma de Kaposi laringeo el paciente, y ante el riesgo de obstrucción de la vía aérea, fue sometido a tratamiento con radiaciones ionizantes sobre PTV-región laringea, mediante dos campos laterales y opuestos, utilizando mascara termolábil de inmovilización y planificación mediante TAC se alcanzó una dosis de 15 Gy a la isodosis del $95 \%$ a un fraccionamiento de $150 \mathrm{cGy}$ /día en 10 fracciones y en 2 semanas. Una semana previa al inicio del tratamiento, durante y una semana después de haber finalizado la radioterapia el paciente fue medicado con fluconazol $50 \mathrm{mg}$. V.O./24h.

A las dos semanas de haber finalizado el tratamiento el paciente se había recuperado de su disfonía sin haber presentado toxicidad mucosa aguda y en la actualidad tras un seguimiento de 36 meses, el paciente se haya en remisión completa de su sarcoma de Kaposi laringeo, con controles periódicos. No obstante, en Febrero de 2002 fue diagnosticado de una Giardiasis intestinal, que se resolvió con tratamiento médico y de un Ameboma rectal que requirió intervención quirúrgica que ha dejado como secuelas una incontinencia anorrectal grado IV de caracter irreversible. También ha sido diagnosticado en Abril 2002 de Condilomas acuminados perianales, habiendo sido intervenido en dos ocasiones, en Junio 2002 y Abril 2004. Así mismo en Enero 2003 ha sido diagnosticado de una Hepatopatía crónica por virus $\mathrm{C}$ (informe AP: hepatitis crónica $\mathrm{C}$ de actividad leve, estadio 1 (fibrosis portal); siderosis focal a nivel de células de Kupffer); carga viral VHC (virus hepatitis C) $2.443 .750 \mathrm{UI} / \mathrm{ml}$ genotipo 1, subtipo B. El último estudio de subpoblaciones linfoides en sangre periférica en Enero 2005 muestra los siguientes resultados CD4: 10,3\% (504,7), CD8: 60,7\% (2.974,3), CD3: 76,3, CD4/CD8: 0,17 y carga viral HIV: 304 copias RNA/ml.

\section{Discusión}

El SKE más frecuente por localizaciones es el cutáneo (87\%); las lesiones de cavidad oral, faringe o

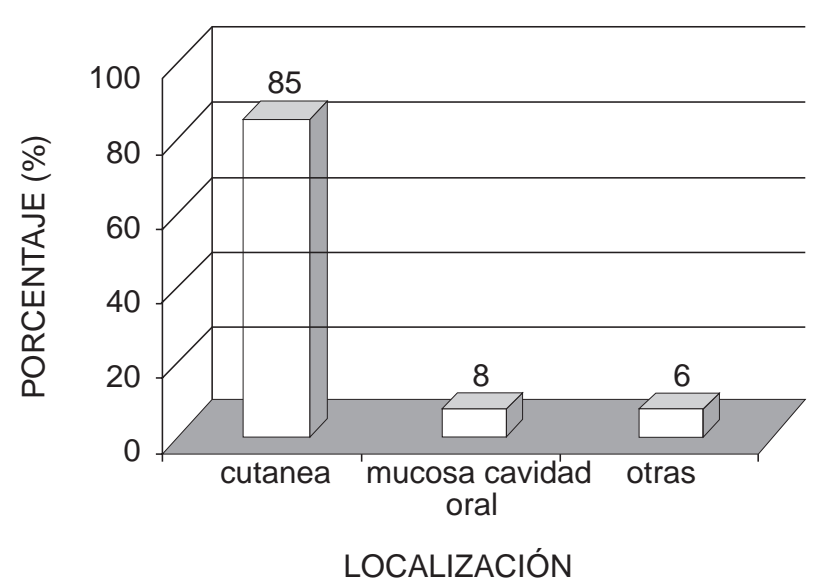

Figura 2. Distribución porcentual por localizaciones del sarcoma de Kaposi epidémico.

laringe son más raras, otros lugares de afectación son la conjuntiva ocular, labios, etc ${ }^{4}$ (Fig. 2). Las lesiones pueden ser de color rosa, violáceo o rojo fuerte y encontrarse en cualquier parte del cuerpo aunque tiene predilección por la parte superior del cuerpo, cabeza y cuello.

Hoy se puede afirmar que la etiopatogenia del SK es consecuencia de una coinfección del virus de la inmunodeficiencia humana (HIV) y del herpes virus humano 8 (HHV 8); el HIV produce una inmunosupresión que facilita la expresión del HHV 8 como $\mathrm{SK}^{5}$.

Aunque la afectación cutánea, la afectación visceral y ganglionar son más frecuentes en el SKE que en la forma "clásica", el SKE no es generalmente la principal causa de muerte en los pacientes con SIDA, quienes fundamentalmente fallecen por otro tipo de "desordenes", por lo general infecciones oportunistas. Sin embargo, el SKE ocasiona muchos problemas para pacientes que están en fases relativamente precoces del SIDA, entre estos se incluyen alteraciones cosméticas, dolor y compresiones secundarios al crecimiento del tumor, que disminuyen la calidad de vida de estos pacientes ${ }^{1}$.

La incidencia de SK como signo inicial del SIDA ha descendido desde aproximadamente un $50 \%$ al comienzo de los años $80^{6}$ hasta un $14 \%$ al comienzo de $\operatorname{los} 90^{7,8,9}$. La explicación para este descenso se encuentra en la existencia de otros grupos de riesgo (p.e. adictos a drogas por vía parenteral), cambios en los criterios de clasificación del SIDA o cambios 
en los comportamientos sexuales de la población homosexual, pero la causa más importante para esta disminución de la incidencia ha sido sin lugar a dudas la introducción del HAART ${ }^{10}$.

En este sentido dado que el SKE suele tener una distribución multifocal en el momento del diagnóstico, se debe considerar como mejor opción terapéutica un tratamiento sistémico. Y dentro del tratamiento sistémico se pueden establecer diferentes alternativas.

La utilización del HAART en estos pacientes no sólo ha dado como resultado una estabilización de las lesiones existentes o un enlentecimiento en el crecimiento y progresión de la enfermedad ${ }^{11}$, sino que consigue incluso una disminución mensurable de las lesiones existentes ${ }^{12}$. Algunos estudios correlacionan esta respuesta clínica con la disminución en plasma de los niveles de RNA HIV-1, un incremento de los niveles de linfocitos CD4+ y una distinción de los anticuerpos HHV-8 ${ }^{13}$. Otros más recientes postulan que la respuesta inmune podría contribuir a la disminución de la incidencia de SKE durante la administración del HAART, pero es improbable que justifique de forma completa la rápida y frecuente resolución del SKE cuando se inicia el HAART $^{14}$.

El tratamiento con interferones ha sido utilizado durante muchos años en el tratamiento del SKE. En los primeros ensayos clínicos, los interferones tenían una acción antiviral, antiproliferativa, así como inmunomoduladora ${ }^{15}$. Las respuesta clínica al interferón alfa (es un inhibidor in vitro del HIV y también tiene actividad antiangiogénica) cuando se utilizaba como agente único está condicionada por el estado inmune del paciente. Así pacientes con cifras de CD4 $\geq 400 / \mu \mathrm{L}$ era más probable que respondieran que aquellos con valores de CD4 $<150 / \mu \mathrm{L}^{16}$.

El tratamiento con quimioterapia (QT) sistémica se debe aplicar a aquellos pacientes con enfermedad sintomática y que no puedan ser tratados con interferón alfa. No obstante dado el estado inmunitario de estos pacientes se deben considerar protocolos de quimioterapia con disminución de la dosis habitual para evitar morbilidad. Dentro de los esquemas de quimioterapia sistémica se debe considerar como recomendado el formado por una asociación de adriamicina (ADR), bleomicina (BLE) y vincristina $(\mathrm{VCR})^{17}$. Más recientemente la utilización de antraciclinas liposomiales ${ }^{18}$ en estudios randomizados, comparándolas al esquema de $\mathrm{ABV}$, han demostrado que su eficacia es la misma pero con menor toxicidad. Las respuestas a estos tratamientos sistémicos son frecuentemente parciales y temporales y la tolerancia es variable particularmente si se considera la toxicidad hematológica. Otras alternativas serían, cuando existen resistencia a los agentes liposomiales el empleo de paclitaxel o VP-16 ${ }^{19}$. El empleo de quimioterapia y HAART puede hacer que la administración de QT se pueda hacer de manera discontinua.

En ocasiones los pacientes no requieren tratamientos sistémicos, sino locales.

La opción de escisión quirúrgica de la lesión es muy rara. Otras alternativas son la crioterapia (con nitrógeno líquido), inyecciones intralesionales de vinblastina (VBL), o de la hormona gonadotropina coriónica humana (hCG) o más recientemente aplicaciones tópicas de un gel de alitretinoína (ácido 9-cisretinoico).

La Radioterapia es efectiva y beneficiosa como terapia local, consiguiendo una respuesta satisfactoria en el $90 \%$ de los casos tratados. Las lesiones en la cavidad oral son la localización mucosa más frecuente de SKE, 51\% según Lozada et $\mathrm{al}^{4}$. En general la radioterapia ofrece un efecto paliativo en muchos casos, no obstante, se deben tener en cuenta las toxicidades que puede ocasionar. El mayor problema que presentan estos pacientes cuando son sometidos a RT es la toxicidad aguda en forma de mucositis $^{20}$, sin embargo, no todos los autores han observado mucositis severas (grado 3, clasificación RTOG), en pacientes con SIDA que hubiesen recibido irradiación sobre cavidad oral, laringe o faringe. Chak et al..$^{21}$ habla de la existencia de reacciones agudas severas en pacientes que recibieron una dosis superior a 16 Gy y que estas disminuyeron, en cuanto a su severidad, al bajar las dosis a $14 \mathrm{~Gy}$. Por tanto una dosis de 15 Gy sería suficiente para producir una disminución del tumor y conseguir una buena paliación de los síntomas sin gran toxici$\mathrm{dad}^{22}$.

Considerando que los pacientes con SIDA padecen infecciones oportunistas Le Bourgeois et al. ${ }^{23}$ sugiere que una candidiasis subclínica podría ser responsable del incremento de la toxicidad aguda cuando se irradian estos pacientes, no obstante, el tratamiento antifúngico local y sistémico profiláctico no siempre previene la mucositis ${ }^{24}$. 


\section{Bibliografia}

1. Geara F, Le Bourgeois JP, Piedbois P, et al. Radiotherapy in the management of cutaneous epidemic Kaposi's sarcoma. Int. J. Radiat. Oncol. Biol. Phys 1991; 21: 1517-1522.

2. Berral V, Peterman TA, Berkelman R L, et al. Kaposi's sarcoma among persons with AIDS. a sexually transmitted infection? Lancet 1990; 335: 123-128.

3. Munro AJ, Stewart JS. AIDS: Incidence and managament of malignant disease. Radiother. Oncol 1989; 14: 121-131.

4. Lozada F, Silverman S, Migliotari CA, et al. Oral manifestations of tumor and opportunistic infections in the acquired immunodeficiency syndrome (AIDS): findings in 53 homosexual men with Kaposi`s sarcoma. Oral Surg 1983; 56: 491-494.

5. Gao SJ, Kingsley L, Hoover DR, et al. Seroconversion to antibodies against Kaposi's sarcoma-associated herpesvirus-related latent nuclear antigents before the development of Kaposi's sarcoma. N Engl J Med 1996; 335: 233-241.

6. DeJarlais DC, Marmor M, Thomas P, et al. Kaposi's sarcoma among four different AIDS risk groups. N Engl J Med 1984; 310: 1119 .

7. Gallant JE, Moore RD, Richman DD, et al. Risk factors for Kaposi's sarcoma in patients with advanced human immunodeficiency virus disease treated with zidovudine. Zidovudine Epidemiology Study Group. Arch Intern Med 1994; 154: 566-572.

8. International Collaboration on HIV and CancerHigghly avtive antiretroviral therapy and incidence of cancer in human immunodeficiency virus-infected adults. J Natl Cancer Inst 2000; 92: 1823-1830.

9. Ledergerber B, Egger M, Erard V, et al. AIDS-related opportunistic illnesses ocurring after initiation of potent antiretroviral therapy: the Swiss Cohort Study. JAMA 1999; 282: 2220-2226.

10. Lebbe C, Blum L, Pellet C, et al. Clinical and biological impact of antiretroviral therapy with protease inhibitors on HIV-related Kaposi's sarcoma. AIDS 1998; 12: 45-49.

11. Bower M, Fox P, Fife K, et al. Highly active anti-retroviral therapy (HAART) prolongs time to treatment failure in Kaposi's sarcoma. AIDS 1999; 13: 2105-2111.

12. Cattelan AM, Calabro ML, Aversa SM, etal. Regression of AIDS-related Kaposi's sarcoma following antiretroviral therapy with protease inhibitors: biological correlates of clinical outcome. Eur J Cancer 1999; 35: 1809-1815.
13. Dupin N, Rubin de Cervens V, Gorin I, et al. The influence of highly active antiretroviral therapy on AIDS-associated Kaposi's sarcoma. Br J Dermatol 1999; 140: 875-881.

14. Bourboulia D, Aldam D, Lagos D, et al. Short and long term effects of highly active antiretroviral therapy on Kaposi sarcoma-associated herpesvirus immune responses and viraemia. AIDS 2004; 20: 485-493.

15. Tulpule A, Joshi B, DeGuzman N, et al. Interleukin-4 in the treatment of AIDS-related Kaposi's sarcoma. Ann Oncol 1997; 8: 79-83.

16. Lane HC, Kovacs JA, Feinberg J, et al. Anti-retroviral effects of interferon-alpha in AIDS-associated Kaposis's sarcoma. Lancet 1988; 2: 1218-1222.

17. Gill PS, Rarick MU, McCutchan JA, et al. Systemic treatment of AIDS-related Kaposi's sarcoma: results of a randomized trial. Am J Med 1991; 90: 427-433.

18. Nuñez M, Saballs PValencia ME, et al. Response to liposomal doxorubicin and clinical outcome of HIV-1-infected patients with Kaposi's sarcoma receiving highly active antiretroviral therapy. HIV Clin Trials 2001; 2: 429-437.

19. Stebbing J, Wildfire A, Portsmouth S, et al. Paclitaxel for anthracycline-resistant AIDS-related Kaposi's sarcoma: clinical and angiogenic correlations. Ann Oncol 2003; 14: 1660-1666.

20. Le Bourgeois JP, Frikha H, Piedbois P, et al. Radiotherapy in the management of epidemic Kaposi's sarcoma of the oral cavity, the eyelid and the genitals. Radiother Oncol 1994; 30: 263-266.

21. Chak, L.; Gill, P.S.; Levine, A.M.; Meyer, A.M.; Anselmo, J.A.; Petrovitch, Z. Radiation therapy for acquired immunodeficiency syndrome-related Kaposi`s sarcoma. J. Clin. Oncol 1988; 6:863-867.

22. Kirova YM, Belembaogo E, Frikha H, et al. Radiotherapy in the management of epidemic Kaposi's sarcoma: a retrospective study of 643 cases. Radiother Oncol 1998; 46: 1922.

23. Piedbois P, Frikha H, Martin L, et al. Radiotherapy in the management of epidemic Kaposi's sarcoma. Int J Radiat Oncol Biol Phys 1994; 30: 1207-1211.

24. Kao GD, Devine P, Mirza N. Oral cavity and oropharyngeal tumors in human immunodeficiency virus-positive patients: acute response to radiation therapy. Arch Otolaryngol Head Neck Surg 1999; 125: 873-876.
Correspondencia:

Dr. F. J. Andreu Martínez

Servicio de Oncología Radioterápica

Hospital Universitari Sant Joan

Crta. Ncal. 332 Alicante-Valencia s/n

E-03550 Sant Joan d'Alacant (Alicante)

E-mail: andreu_fra@gva.es. 